\title{
Der Alpenrhein - Versuch einer nachhaltigen Entwicklung für den größten alpinen Wildfluss
}

The Alpine Rhine - An attempt at sustainable development for the largest torrential river in the Alps

\author{
von W. Stalzer
}

\section{Kurzfassung/Summary}

Der Charakter des Alpenrheins mit seinem alpinen Einzugsgebiet, seinen Gefällsverhältnissen, seiner Wasserführung und der Schwankungsbreite der Abflüsse prägt diesen als größten alpinen Wildfluss. In den geologischen Zeiträumen hat der Gebirgsabtrag und die folgende Sedimentation zur Ausbildung eines breiten Talbodens zwischen Buchs und der Mündung in den Bodensee geführt. Die Regulierungsmaßnahmen wie zwei Durchstiche, eine Laufverkürzung und Eindämmungen haben eine sehr intensive Nutzung dieser Region ermöglicht. Die Forderung nach Anhebung des Schutzzieles von $\mathrm{HQ}_{100}$ auf $\mathrm{HQ}_{300}$ ist in Anbetracht des enormen Schadenspotentiales verständlich, aber auch ein Abbau der mit der seinerzeitigen Regulierung und der Wasserkraftnutzung verbundenen Landschafts- und Ökologiedefizite ist ein Gebot unserer Zeit. Die höhenmäßige Sohlfixierung mit der Mündung in den Bodensee in Verbindung mit dem Geschiebeanfall von rd. 2,5 Mio. $\mathrm{m}^{3}$ pro Jahr erfordert zufolge der Verlandung eine Laufverlängerung in den See bzw. ein Geschiebemanagement mit entsprechenden Sedimentationsräumen. Während mit dem Entwicklungskonzept Alpenrhein ein integraler Planungsansatz für die Gestaltung bzw. Nutzung diese Raumes und seiner Tallandschaft und damit aus heutiger Sicht nachhaltiger Lösungsansatz vorliegt, bedingt der Geschiebeeintrag langfristig massive Vorkehrungen in der Raumplanung sowie im Hochwasserschutz und damit ei-

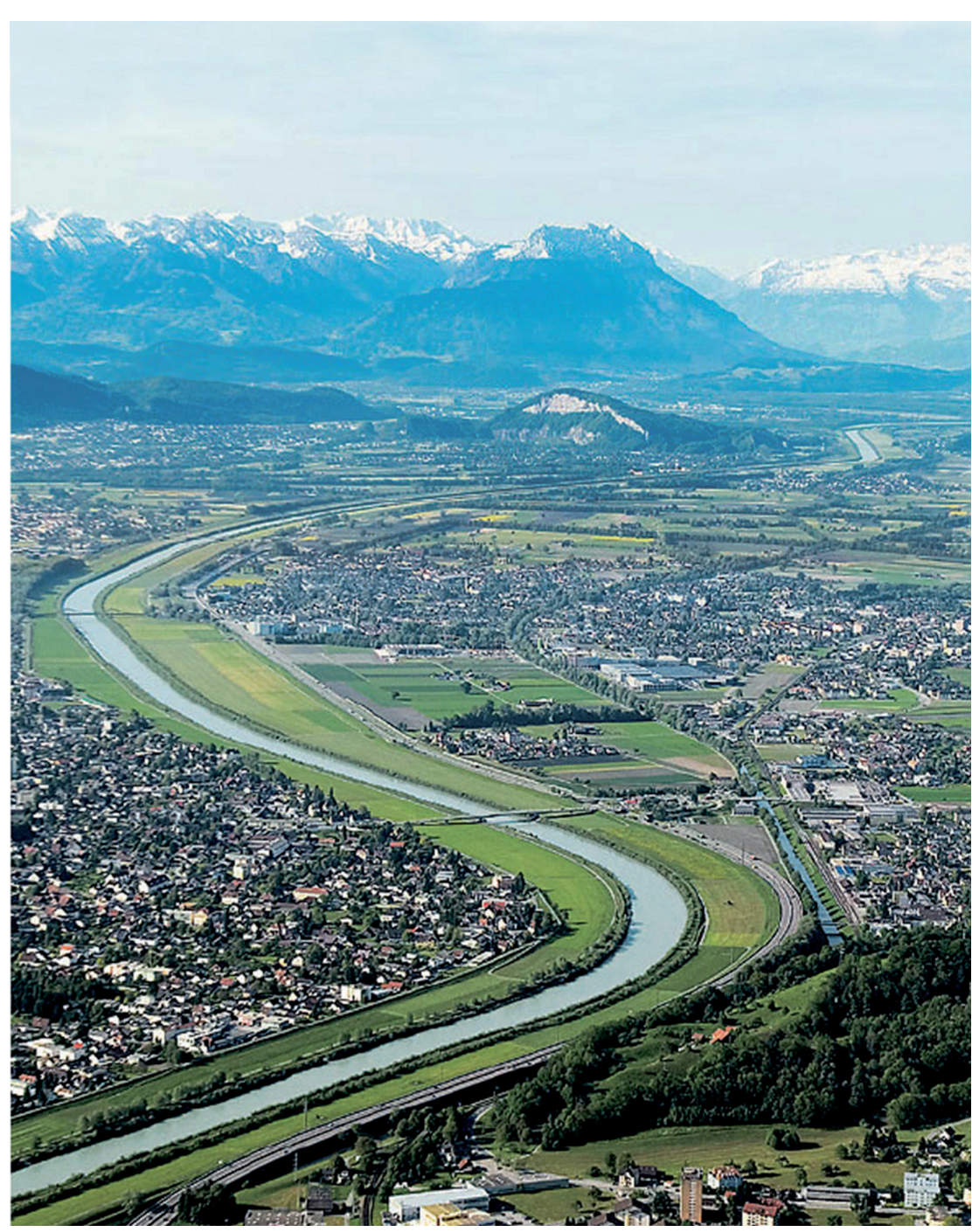

Abb. 1: Besiedelung des Alpenrheintales.

nen ständigen Handlungsbedarf, um das Ungleichgewicht im Gewässersystem aus zu gleichen.

Owing to its special character - the Alpine catchment area, its gradient, its flow and its range of flow variation - the Alpine Rhine is the largest of the torrential rivers in the Alps. Denudation and the resulting sedimentation over geological peri- ods have created a wide valley floor between the town of Buchs (Switzerland, near its border with Austria) and the river's mouth in Lake Constance. Regulation measures such as two cuts, riverbed shortening and dyking have enabled intensive land use to develop in this region. However, while the demand for raising the flood protection target from $H Q_{100}$ to $\mathrm{HQ}_{300}$ is understandable in view of 
the enormous damage potential, the order of the day is to reduce existing damage to landscape and ecology which has been brought about by the regulation of the river and its development for electricity generation. Stabilisation of the bed level at the level of the river's mouth in Lake Constance, combined with an annual bed load transport of about 2.5 million cubic metres, has led to sedimentation, which calls for continuing the river course into the lake or provide for appropriate bed-load management plus the necessary sedimentation spaces. The Alpine Rhine development scheme provides an integrated planning approach for the shaping and utilisation of this region and its valley landscape and, hence, constitutes from today's viewpoint a sustainable solution. In the long term, the substantial bed load transport calls for massive precautions in terms of regional planning and flood protection, and this involves the need for constant action in order to make up for the imbalance in this river system.

\section{Der Rahmen}

Das Einzugsgebiet des Alpenrheins, der heute zwischen Hard und Fußach in den Bodensee mündet, wird hydrologisch in 4 Teilgebiete unterteilt:

- In ein südwestliches Gebiet von $1.514 \mathrm{~km}^{2}$ mit dem Vorderrhein als Hauptgewässer

- In ein südöstliches Gebiet von $1.693 \mathrm{~km}^{2}$ mit dem Hinterrhein als Hauptvorfluter

- In ein Zwischengebiet von Reichenau bis Bad Ragaz mit $1.248 \mathrm{~km}^{2}$, welches von der Landquart der Plessur und der Tamina geprägt ist

- In ein nördliches Gebiet von $1.668 \mathrm{~km}^{2}$ mit der Ill als bestimmendes Gewässer.

Die Quellregionen des Vorderund Hinterrheins in Graubünden mit einem Ausläufer nach Italien bis zu deren Vereinigung zum Alpenrhein in Reichenau können als klassischer Wildbachbereich definiert werden. Bemerkenswert ist hier die hochalpine, teilweise vergletscherte Region mit einer bedeutenden Wasserkraftnutzung, primär über Speicherkraftwerke. Ab dem Zusammenfluss von Vorder- und Hinterrhein und den damit folgenden Alpenrhein bei $\mathrm{km} 0$ folgt bis zum Bodensee eine Fließstrecke von $90 \mathrm{~km}$, die mit ihrem vorherrschenden Gefällsverhältnissen zwischen 2,4 \%o und 1,0 \%o und Fließgeschwindigkeiten im Hochwasserfalle von bis zu $5 \mathrm{~m} / \mathrm{s}$ durch einen Wildflusscharakter geprägt ist. Charakteristisch sind auch die hydrologischen Kennwerte mit dem alpinen Gebietsniederschlag von $1.484 \mathrm{~mm} / \mathrm{a}$ und Abflusshöhen von $1129 \mathrm{~mm}$ bzw. $76 \%$. Gemäß Hydrografischem Jahrbuch für Österreich, 2004, ergaben sich für die Reihe 1971 bis 2004 folgende Jahresabflusswerte:

$\begin{array}{lr}\text { NQT } & 41 \mathrm{~m}^{3} / \mathrm{s} \\ \text { MQ } & 232 \mathrm{~m}^{3} / \mathrm{s} \\ \text { HHQ } & 2.800 \mathrm{~m}^{3} / \mathrm{s}\end{array}$

Gefälle, Abflusscharakteristik und Geologie bedingen gleichzeitig massiveErosionsvorgänge, dieheute an der Mündung mit einer Jahresfracht von rd. 2,5 bis 3,0 Mio. $\mathrm{m}^{3}$ Schwebstoffen und etwa 40.000 $\mathrm{m}^{3}$ Geschiebe zu Buche schlagen. Die Wechselbeziehung von Erosion und Sedimentation war entscheidend für die Ausbildung des Alpenrheintales. Das Sedimentationsverhalten ab dem Bereich von Diepoldsau bedingte eine Sohlhebung mit periodischen Flussverwerfungen, Verfrachtungen in das Umland sowie einen entsprechenden Mündungsvorschub in den Bodensee. Die in geologischen Zeitspannen so entstandenen Talniederungen bildeten gleichzeitig die Grundlage für die Besiedelung und deren wirtschaftlicher Nutzung. Allerdings war insbesondere die Region unterhalb der Illmün- dung stets durch Hochwässer mit einem weitflächigen Abfluss über die Talebene geprägt. Diese Hochwässer selbst, Flussverwerfungen und die verbleibenden Schlammund Kiesablagerungen haben die Bevölkerung Jahrhunderte lang schwerstens bedroht und immer wieder zu katastrophalen Folgen geführt. War die Nutzung dieser Talböden zunächst primär einer intensiveren Landwirtschaft vorbehalten, so folgte mit dem Übergang in das Industriezeitalter ein Wechsel in höherwertige Nutzungen mit einer entsprechender Wirtschaftsgrundlage über Gewerbe, Industrie, Tourismus, Freizeit und so fort. Dementsprechend leben heute im Einzugsgebiet des Alpenrheins über 500.000 Menschen, einer Region, die einen blühenden Wirtschaftsstandort darstellt und ein hohes Potenzial für attraktive Freizeit und Naherholung sowie artenreiche Lebensräume bietet (Abb. 1). Der prosperierende Lebens- und Wirtschaftsraum vor allem im unteren Rheintal birgt andererseits aber auch ein hohes Schadenspotenzial in sich, das im Falle von Katastrophenereignissen bzw. einem Überschreiten des Bemessungshochwassers $\left(3.100 \mathrm{~m}^{3} / \mathrm{s}\right.$ entsprechend einem $\mathrm{HQ}_{100}$ ) je nach Flutungsszenario bis zu $3 \mathrm{Mrd}$ € beträgt.

\section{Das „Gleichgewicht"}

Im flussbaulichen Sinne wird für unsere Gewässer in der gegebenen Kulturlandschaft eine stabile Sohlenlage angestrebt. Mit dem damit verbundenen konstanten Geschiebehaushalt spricht man von einem „dynamischen“ Gleichgewichtszustand des Gewässers. Der Alpenrhein mit dem eingangs beschriebenen Erosions- bzw. Sedimentationsverhalten zeigte keinesfalls den zitierten Zustand und erforderte massive anthropogene Eingriffe (Regulierungen), um die Sohlhebungen sowie die hochwas- 


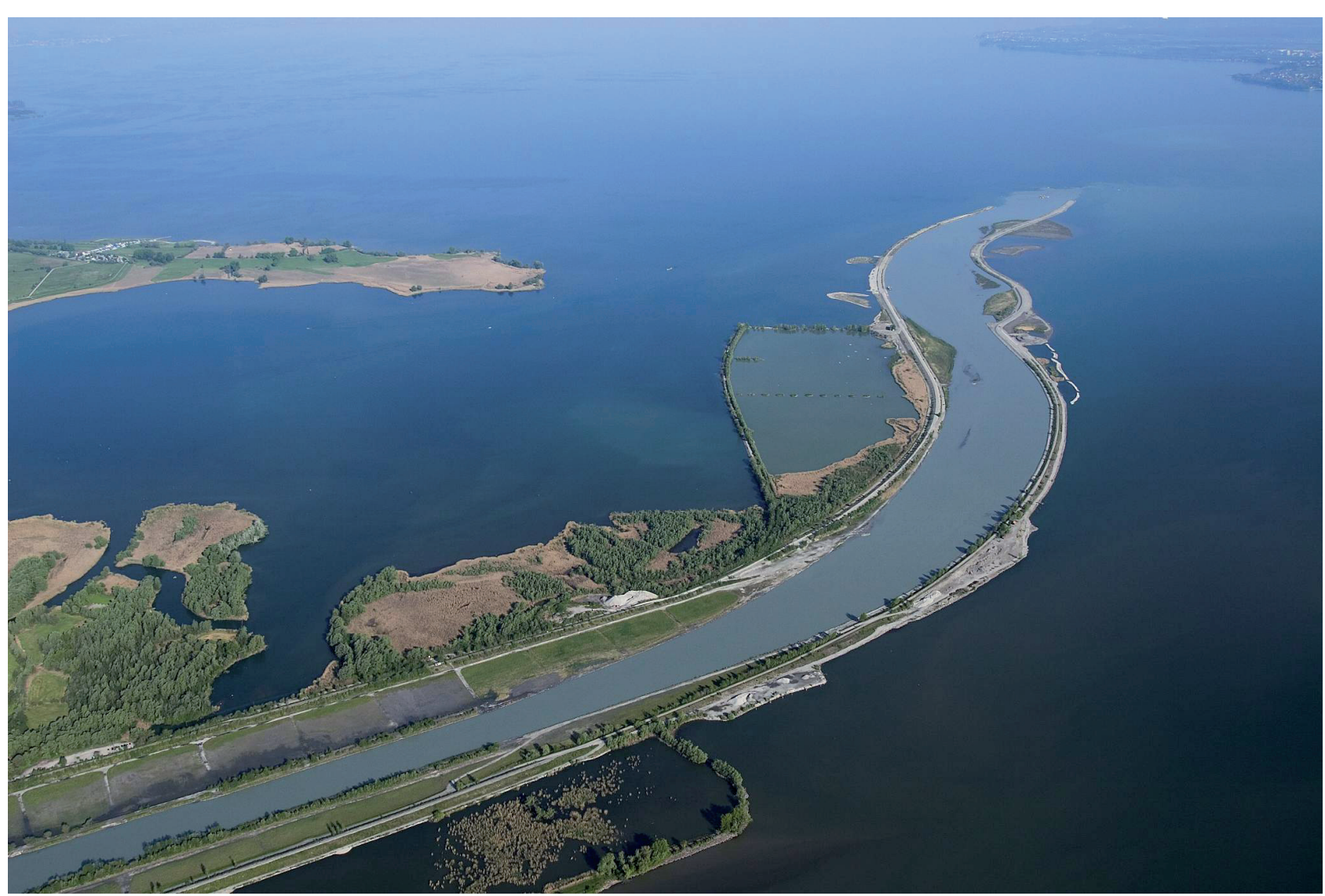

Abb. 2: Mündungsvorstreckung in den Bodensee

serbedingten Verwerfungen und die damit verbundenen Verheerungen hintan zu halten. In einem bilateralen Staatsvertrag wurde daher vor mehr als 110 Jahren eine Regulierung des Rheins zwischen der Illmündung und dem Bodensee auf der so genannten internationalen Strecke beschlossen. Primäres Ziel der gemeinsam getragenen Maßnahmen bildete der Hochwasserschutz bis zu einem definierten Abfluss von $3.100 \mathrm{~m}^{3} / \mathrm{s}$. Mittels der Durchstiche bei Diepoldsau und Fußach wurde der Lauf der hier vertraglich geregelten Strecke um $10 \mathrm{~km}$ verkürzt und eine Gefällserhöhung auf $1 \%$ o sichergestellt. $\mathrm{Zu}$ Vermeidung weiterer Geschiebeablagerungen wurde der Transportkapazität des neuen Rheinprofils durch die Ausbildung eines Doppelprofiles mit so bezeichneten „Mittelgerinnewuhren“ Rechnung getragen. Häufige Hochwässer mit entsprechendem Geschiebetrieb sollten innerhalb des Mittelgerinnes abgeführt und damit eine übermäßige Verlandung der Vorländer verhindert werden. Bereits bei der laufenden Umsetzung dieses 1. Staatsvertrages aus dem Jahre 1892, der nicht nur die zitierten Durchstiche, sondern auch entsprechende Maßnahmen zur Binnengewässerausleitung und zum Geschieberückhalt im Einzugsgebiet regelte, wurde die Notwendigkeit einer Vertragsergänzung deutlich. Aufgrund der zunehmenden Verlandung der Fussacher-, Harder- und Bregenzerbucht wurde 1924 ein weiterer Staatsvertrag abgeschlossen, der neben der Fortführung der Regulierungsarbeiten auch die Vorstreckung der Regulierungsbauwerke auf dem Schuttkegel (Halde) im Bodensee vorsah. Mit letzterer Maßnahme sollte die Geschiebe- und Feststofffracht in die tieferen Seebereiche eingeleitet werden. In weiterer Folge ließen zudem die Geschiebeablagerungen im Mittelgerinne erkennen, dass die Breite mit ursprünglich $110 \mathrm{~m} \mathrm{zu}$ groß gewählt worden war. Auf Basis entsprechender Untersuchungen der Versuchsanstalt für Wasserbau, Hydrologie und Glaziologie (VAW) der Eidgenössischen Technischen Hochschule (ETH) Zürich wurden entsprechende Lösungsvorschläge zur Gerinnegestaltung erarbeitet, die Anlass für einen dritten und bisher letzten Staatsvertrag im Jahre 1954 waren. Festgelegt wurden damit

- eine Erhöhung der Mittelgerinnewuhre von der Illmündung bis zum Bodensee bei gleichzeitiger Einengung des Mittelgerinnes selbst;

- eine Verstärkung und Zurücksetzung der Hochwasserdämme für 


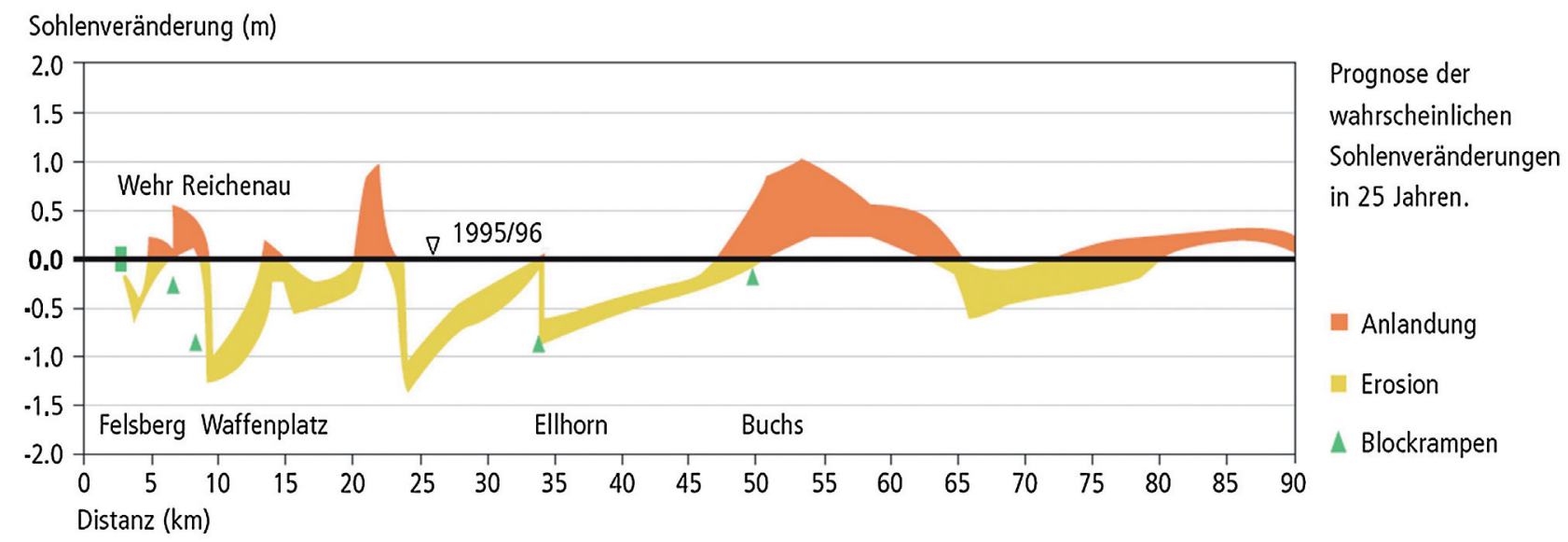

Abb. 3: Prognostizierte Sohlentwicklung

eine Hochwasserabflussmenge von $3.100 \mathrm{~m}^{3} / \mathrm{s}$ und

- die Weiterführung der Vorstreckung des Rheines in den Bodensee (Abb. 2).

Mit heutigem Stand ist die Umsetzung der Ziele der Staatsverträge weitfortgeschritten. Dievertraglich festgelegte Hochwassersicherheit für ein Bemessungshochwasser von $3.100 \mathrm{~m}^{3} / \mathrm{s}$ ist bei Einhaltung eines Sicherheitsfreibordes von 1 m gewährleistet. Nicht zur Gänze eingehalten werden kann dieser Sicherheitsfreibord jedoch bei zwei nicht in der Zuständigkeit der Internationalen Rheinregulierung (IRR) liegenden Brücken. Dagegen kann die Dammstabilität zwischenzeitlich für nahezu die gesamte Strecke nachgewiesen werden und die Vorstreckung in den Bodensee befindet sich im projektierten Endstadium.

Der über die Regulierungsmaßnahmen erzielte Hochwasserschutz bildete gleichzeitig auch die Grundlage für eine außerordentlich dynamische wirtschaftliche Entwicklung dieser Region und das Rückgrat für die zwischenzeitlich stattgefundene Vervierfachung der Bevölkerungsanzahl seit Umsetzung der Regulierungsmaßnahmen. Die im Staatsvertrag festgelegte statische Zielvorgabe des Hochwasserschutzes mit folgender Übergabe der fertig gestellten Schutzmaßnahmen an die Vertragsstaaten ist heute allerdings nicht realisierbar. In den laufenden Sohlaufnahmen werden je nach örtlichen Randbedingungen Eintiefungen bzw. Auflandungen dokumentiert, die neuerlich „Regulierungseingriffe" erfordern (Abb. 3). Diese Eingriffe sind unter der Maßgabe der Sohlentwicklung und damit des Geschiebehaushaltes auch auf die geänderten gesellschaftspolitischen Vorgaben auszurichten. Vordergründig sind dies das Streben nach einem höheren Hochwasserschutzgrad aber auch Aufgaben zur Verbesserung bzw. Wiederherstellung des Gewässerlebensraumes (ökologischer Zustand) sowie die Anliegen der Raumordnung, des Landschaftsschutzes und des Naturschutzes.

\section{Die Nachhaltigkeit}

Ausgehend von den drei Grundsäulen der Nachhaltigkeit ergeben sich für den Alpenrhein und sein Einzugsgebiet folgende Handlungsschwerpunkte:

Sozialer-gesellschaftspolitischer Bereich

- Absicherung der Lebens- und
Wirtschaftsgrundlagen und notwendigen Entwicklungsmöglichkeiten

- Verfügbarkeit über die Ressourcen (Trinkwasser, Brauchwasser, Rohstoffe etc.)

- Schutz des Menschen und seines Lebensraumes, Erholungs- und Freizeitraumes, Tourismus

Ökonomischer Bereich

- Schutz und Entwicklung des Wirtschaftsraumes

- Absicherung der Entwicklung von Landwirtschaft, Gewerbe und Industrie sowie Dienstleistung

- Sicherstellung der Infrastruktur

- Entwicklung regionaler Wirtschaftschwerpunkte wie Wasserkraft etc.

Ökologiebereich

- Lebensraum für den aquatischen und den terrestrischen Bereich

- Gewässerschutz

- Grundwasser

- Raum- und Flächenverlust etc.

Die besondere Herausforderung liegt nunmehr in der Erstellung eines abgestimmten integralen Gesamtkonzeptes für die Region Alpenrhein. Eine Aufgabe, die von der Gemeinsamen Rheinkommission (GRK), der IRR sowie von der Internationalen Regie- 
Strategie/Szenario

Grundsätzliche Möglichkeiten

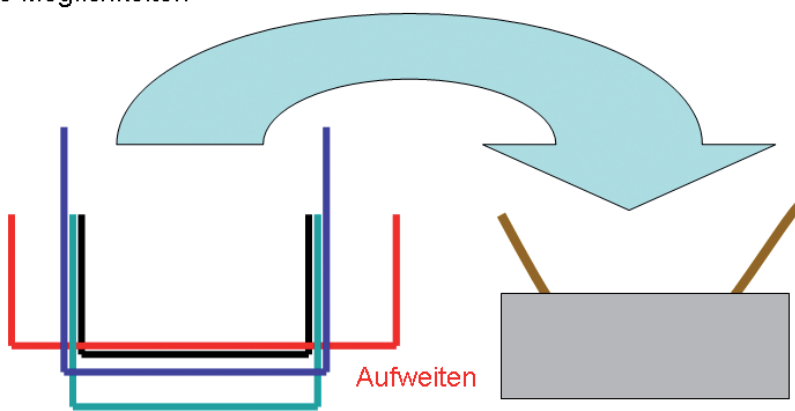

Eintiefen

Erhöhen

Ausleiten

Speichern

Abb. 4: Systemskizze zur Erhöhung der Abflusskapazität auf $\mathrm{HQ}_{300}$

rungskommission Alpenrhein (IRKA) gemeinsam bearbeitet wurde und deren Ergebnisse im Entwicklungskonzept Alpenrhein 2005 der Öffentlichkeit vorgestellt wurden. Neben einer generellen Entwicklungsstrategie wurde ein konkretes Maßnahmenkonzept erarbeitet, das eine vernetzte $\mathrm{Ab}$ deckung der drei Handlungsfelder Hochwasserschutz, Grundwasser und Gewässerökologie ermöglichen soll.

Als Maßnahmenpakete wurden folgende Lösungsansätze zusammengefasst:

- Aufweitungen und Geschiebemanagement mit Hilfe von Querschnittserweiterungen und damit Erhöhung der Abflusskapazität im Bereich der internationalen Strecke sowie einer Bettstabilisierung in Eintiefungszonen (Abb. 4);

- Vernetzung der Gewässersysteme (Anschluss der Nebengewässer) und Längsdurchgängigkeit als entsprechende Priorität im Ökologiebereich;

- Lösung der Schwallproblematik über Ausgleichsspeicher bzw. angepasste Betriebsweisen zur Wiederherstellung flusstypspezifischer Lebensräume;

- Entwicklung der Wasserkraft unter Beachtung der ökologischen Rahmenbedingungen;
- Sicherung der rheinnahen Gebiete für eine Gewässerentwicklung und einen künftigen Handlungsspielraum für Schutzmaßnahmen sowie eine mögliche Ressourcennutzung und für die Naherholung;

- Berücksichtigung eines Überlastfalles und Notentlastung in nutzungsextensivere Bereiche.

\section{Realität und Vision}

Die Umsetzung des Entwicklungskonzeptes Alpenrhein wird zumindest mehrere Jahrzehnte in Anspruch nehmen und einer dynamischen Anpassung bedürfen. Alleine eine Erhöhung des Schutzzieles von derzeit $3.100 \mathrm{~m}^{3} / \mathrm{s}$ auf ein $\mathrm{HQ}_{300}$ mit einem Bemessungsabfluss $4.300 \mathrm{~m}^{3} / \mathrm{s}$ erfordert nicht nur entsprechende Planungs-, Versuchs- und Umsetzungszeiträume sondern vor allem neben der Akzeptanz seitens der Betroffenen auch die Bereitstellung der notwendigen finanziellen Mittel in Höhe von zumindest 300 bis 500 Mio. $€$. Verfolgt man hiezu vergleichsweise die Gewässerentwicklung der vergangenen 100 Jahre, so stellt sich automatisch die Frage „Quo vadis Alpenrhein in den nächsten 100 Jahren?“. Als Kardinalpunkte derartiger Visionen sind neben den sozioökono- mischen Aspekten wie der Bevölkerungsentwicklung mit einem Zuwachs um $50 \%$ innerhalb der letzten 30 Jahre, einer Verdoppelung des Flächenbedarfs für Bauland sowie Infrastruktur und einer Verdreifachung des Energiebedarfes vor allem die naturräumlichen Vorgaben, die da lauten Geschiebe- und Schwebstoffhaushalt sowie Klimawandel, anzusehen.

Die damit heute erkennbaren Szenarien wurden anlässlich eines Workshops „Alpenrhein 2100“ im Jahre 2006 umrissen und berücksichtigten vor allem das gesellschaftlich artikulierte Schutzbedürfnis für den Lebens- und Wirtschaftsraum in Verbindung mit dem naturbedingten bzw. anthropogen beeinflussten Langzeitaspekt.

Die Querbeziehung und damit Vernetzung der hier maßgebenden Parameter kann nur beispielhaft aufgezeigt werden:

Hochwasserschutz

- Niederschlags-Abflussverhalten

- Gefährdungspotenzial

- Flächennutzung und -verfügbarkeit

Hydrologie und Geschiebehaushalt

- Klima

- Erosions- und Sedimentationsverhalten

- Flächenverfügbarkeit

- Transportmechanismen

Wirtschaftsraum

- Infrastruktur

- Flächenverfügbarkeit

- Ressourcenverfügbarkeit

- Schutz vor Naturgefahren

Erholung und Tourismus

- Wertewandel im Bedarf

- Flächenverfügbarkeit

- Erlebnis und Naturraum

Landschafts- und Naturschutz

- naturnahes Raumpotenzial

- Habitatansprüche

- aquatischer und terrestrischer Lebensraum 


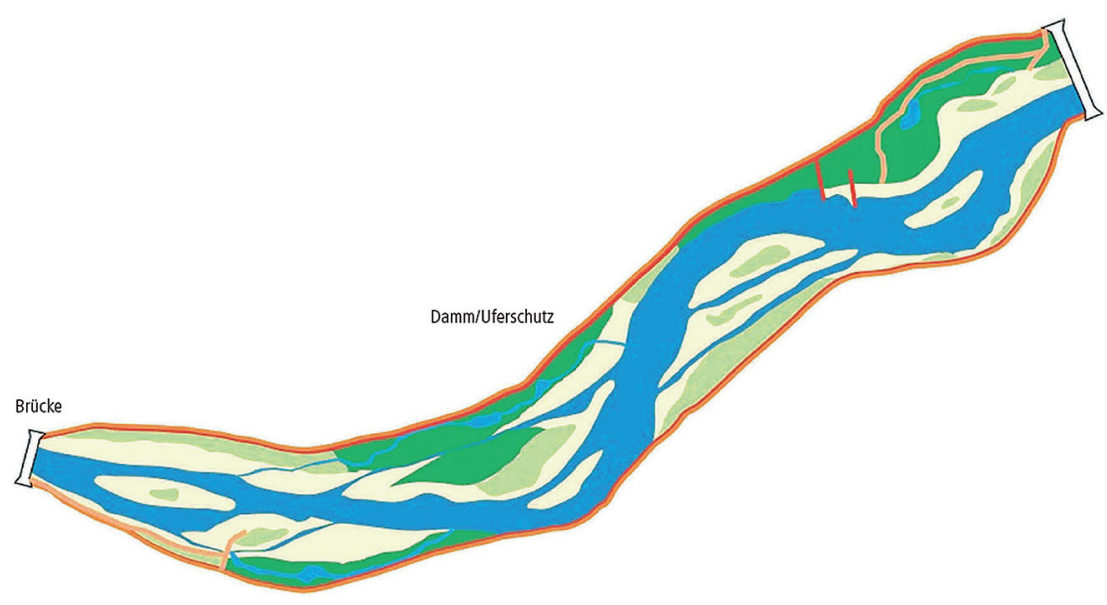

Abb. 5: Systemskizze Aufweitung
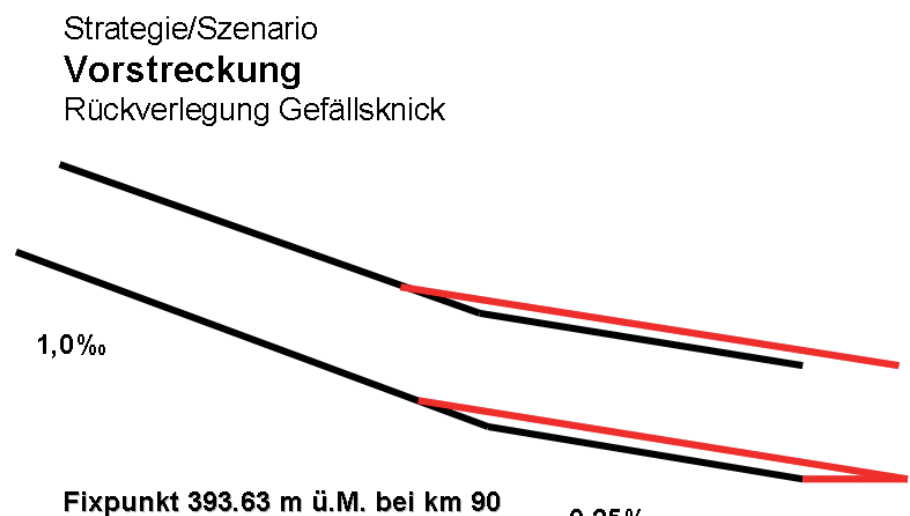

$0,25 \%$

Abb. 6: Systemskizze Lösungsansatz für Sedimentverlagerung in den Bodensee

Ansprüche der Gesellschaft

- Sicherheit, Lebensgrundlagen

- wirtschaftliche Entwicklung

- Erholung - Freizeit - Gesundheit

- Entscheidungsfreiheit - BürgerInnenbeteiligung

Mit dieser Aufzählung kann nur ein sehr beschränkter Themenkatalog gestreift werden, klar kristallisieren sich jedoch zwei Hauptmomente und zwar:

- der vorbeugende Hochwasserschutz und

- eine entsprechende Flächenverfügbarkeit bzw. Umsetzung in der Raumplanung

heraus.
Der ExpertInnenkreis des Workshops 2100 hat daher nach einer visionären Bearbeitung Lösungsansätze und zugehörige Prioritäten artikuliert. Eine Aufarbeitung der Thematik des vorbeugenden Hochwasserschutzes erfordert neben der heute aktualisierten Frage des Schutz- bzw. Ausbaugrades mit der angesprochenen Steigerung auf $4.300 \mathrm{~m}^{3} / \mathrm{s}$ kurzfristig die Ausarbeitung eines Notfallkonzeptes für den Überlastfall mit den schon diskutierten Entlastungsflächen bzw. -korridoren. Parallel dazu sind die Möglichkeiten einer Erhöhung der Abflusskapazität mit den Kernvarianten einer Bettaufweitung innerhalb der Hochwasserdämme bzw. von Dammerhöhungen sowie gegebenenfalls auch Kombinationen beider hinsichtlich deren Machbarkeit und Langzeitverhalten $\mathrm{zu}$ untersuchen (Abb. 5). In Zusammenhang mit dem angesprochenen Betrachtungszeitraum ist der Querbezug Geschiebehaushalt, Geschiebetransportverhalten und Sohlgefälle aufzuarbeiten. Die heute gehandhabte Verlagerung von rd. 2,5 bis 3 Mio. $\mathrm{m}^{3}$ Feststoffen in die Tiefenzonen des Bodensees erfordert eine Lenkung dieses „Feststoffstromes“ auf der Halde, die so genannte Vorstreckung. Bei dem gegebenen "Höhenfixpunkt“ des Bodensee-Wasserspiegels bedingt derzeit jeder Kilometer Vorstreckung bei dem erforderlichen Gefälle eine Aufhöhung um 0,25 m. Würde die aufgelandete Halde sich so in den See weiter vorschieben wie in den vergangenen 100 Jahren, so wäre eine weitere Verlängerung um 5 Kilometer mit einer Sohlhebung bei km 90, dem Beginn der Vorstreckung, um 1,25 m erforderlich. Die so zu erwartende rückschreitende Sohlhebung bedingt gleichzeitig eine Abflussprofilverkleinerung und damit eine Kapazitätsminderung. Mit einer Rückverschiebung des heute bei km 90 gegebenen Gefällsknickes von $1 \%$ auf $0,25 \%$ o um $1,50 \mathrm{~km}$ und gleichzeitiger Erhöhung der Vorstreckungsdämme um 1,25 m wäre hier der notwendige Ausgleich sichergestellt (Abb. 6). Auch eine derartige Maßnahme bedeutet somit keine dauerhafte Lösung der Auflandungs- bzw. Sedimentationsproblematik. Darüber hinaus bleibt die Aufgabe einer gezielten Kiesentnahme wie sie derzeit mit rd. $40000 \mathrm{~m}^{3} / \mathrm{Jahr}$ in der Vorstreckung erfolgt. Eine Maßnahme, die auch künftig bei diversen Profilaufweitungen mit entsprechendem Einfluss auf die Schleppkraft erforderlich sein wird. Die Dauerbelastung aus dem Gebirgsabtrag mit 2,5 bis 3 Mio. $\mathrm{m}^{3}$ jährlicher Feststofffracht stellt somit 
die naturräumliche Grundproblematik dar. Auf 100 Jahre bezogen entspricht dies einem Raumbedarf von 250 Mio. $\mathrm{m}^{3}$ bzw. einer Fläche von $50 \mathrm{~km}^{2}$ mit einer Aufhöhung um $5 \mathrm{~m}$. Dieser „Landgewinnung“ muss zwangsläufig mehrdimensional (horizontal aber auch vertikal) sowie auch aufgefächert Rechnung getragen werden. Angeboten wurde u.a. eine Auflandung bestimmter Talniederungsbereiche in Verbindung mit einer Verbreiterung des Mündungsdeltas, aber auch eine Sedimentationslenkung im Sinne von Inselbildungen im Bodensee selbst. Zwangsläufig verbunden mit dieser Kernthematik sind die Frage der Flächenverfügbarkeit und der Nutzungsverschiebungen sowie die Auseinandersetzungen bezüglich der gegensätzlichen Ansprüche von Siedlungsraum, Gewässer und Naturschutz. Ein Beharren auf dem IST-Zustand be- deutet in diesem Falle Rückschritt bei gleichzeitiger Gefährdungszunahme.

Im Resumeé ist daher festzuhalten, dass sich für die Sohle des Alpenrheins zufolge der naturräumlichen Vorgaben kein dynamisches Gleichgewicht einstellen kann. Eine Kombination von Hochwassermanagement, Geschiebemanagement und Flächenmanagement (Raumplanung) wird stets anthropogene Eingriffe erfordern. Eine Nachhaltigkeit für den Alpenrhein im Sinne des Ausgleiches der ökonomischen, ökologischen und sozialen Komponenten in Abhängigkeit der jeweiligen gesellschaftspolitischen Ansprüche ist daher jedenfalls anzustreben und erfordert sich stets anpassende anthropogene Reaktionen.

Eine Nachhaltigkeit im Sinne einer unveränderten Ressourcenverfügbarkeit für künftige Genera- tionen ist nicht gegeben. In diesem Sinne sind hier die Kernaussagen der ExpertInnen des Workshops Alpenrhein $2100 \mathrm{zu}$ wiederholen:

- Einen absoluten Schutz vor Naturgefahren gibt es nicht.

- Nicht Landschaft konservieren, sondern sich für Nutzungs- und Geländeveränderungen in den nächsten 100 Jahren öffnen.

\section{LITERATUR}

--- (1992): Der Alpenrhein und seine Regulierung, Internationale Rheinregulierung, 1892-1992; Rorschach; Buchsdruck und Verlag --- (2005): Entwicklungskonzept Alpenrhein Kurzbericht; Internationale Regierungskommission Alpenrhein und Internationale Rheinregulierung

Stalzer, Wolfg.: (2007) Alpenrhein 2100, vom Gestern zum Morgen im Alpenrheintal; Osterrei59.Jg. SpringerWienNewYork

Korrespondenz:

Dipl. Ing. Dr. Wolfgang Stalze Weimarerstraße 78

1180 Wien 